\title{
Alan Dundes, Two Tales of Crow and Sparrow. A Freudian Folkloristic Essay on Caste and Untouchability
}

Lanham, Rowman \& Littlefield Publishers, 1997, XII + 163 p., bibl.

\section{Gilles Tarabout}

\section{OpenEdition}

\section{Journals}

Édition électronique

URL : http://journals.openedition.org//homme/8115

DOI : 10.4000//homme.8115

ISSN : 1953-8103

Éditeur

Éditions de l'EHESS

Édition imprimée

Date de publication : 1 janvier 2002

Pagination : 275-276

ISBN : 2-7132-1404-1

ISSN : 0439-4216

\section{Référence électronique}

Gilles Tarabout, «Alan Dundes, Two Tales of Crow and Sparrow. A Freudian Folkloristic Essay on Caste and Untouchability », L'Homme [En ligne], 161 | janvier-mars 2002, mis en ligne le 06 juin 2007, consulté le 24 septembre 2020. URL : http://journals.openedition.org//homme/8115 ; DOl : https://doi.org/ 10.4000/lhomme.8115

Ce document a été généré automatiquement le 24 septembre 2020

(c) École des hautes études en sciences sociales 


\title{
Alan Dundes, Two Tales of Crow and Sparrow. A Freudian Folkloristic Essay on Caste and Untouchability
}

Lanham, Rowman \& Littlefield Publishers, 1997, XII + 163 p., bibl.

\author{
Gilles Tarabout
}

1 ALAN DUNDES, professeur d'anthropologie et de folklore à l'Université de Berkeley depuis quarante ans, a développé une approche psychanalytique des contes, lesquels constituent selon lui une sorte "d'ethnographie autobiographique, une image d'un peuple peinte par ce peuple (par opposition aux portraits dessinés par des observateurs externes soi-disant objectifs) »(p. Ix). Ce livre applique ce principe au domaine indien, dont l'auteur reconnaît ne pas être spécialiste.

2 Le propos de l'ouvrage est d'«expliquer " le système des castes et la pratique de l'intouchabilité en Inde. L'auteur s'appuie sur deux contes indiens et leurs variantes, mettant en scène un moineau et un corbeau. Montrant que ces histoires présentent, par comparaison avec d'autres du même genre ailleurs dans le monde, des particularités propres à l'aire culturelle considérée, il en propose une herméneutique ("l'ethnographie autobiographique " nécessite malgré tout son interprète). Celle-ci, sur la base d'une assimilation (en effet plausible) entre moineau et brahmane d'une part, corbeau et intouchable de l'autre, lui permet de mettre en évidence dans le premier conte l'expression d'une immutabilité de la structure sociale, et dans le second la manifestation d'un lien privilégié entre intouchabilité et excréments. L'essentiel du livre est consacré à développer ce dernier point.

3 On le sait, la plupart des auteurs qui ont traité de la question de l'impureté en Inde, impureté qui, au-delà des controverses théoriques, est au cœur des rapports concrets entre castes, ont envisagé son possible fondement dans les diverses "productions " corporelles - poils, ongles, salive, sueur, mucus, sang, semen, urine, fèces - ou dans la mort - impureté du cadavre. Alan Dundes considère que toutes ces impuretés se ramènent à une, la seule à être véritablement "explicative » : celle des excréments. Cela le conduit d'ailleurs curieusement à « décorporéiser » quelque peu les matières 
fécales et à les intégrer à la théorie brahmanique des «restes » afin de procéder à un certain nombre d'autres généralisations.

4 Le système des castes, et plus spécialement la pratique de l'intouchabilité, découlent donc d'une fixation anale (je résume). Encore faut-il rendre compte de cette dernière. L'auteur en localise les causes dans l'acquisition de la propreté par le petit enfant. D'après les sources consultées, celle-ci pourrait paraître de prime abord peu traumatisante en Inde. Alan Dundes fait néanmoins valoir qu'elle commence très tôt (trois mois), et que la période de bienveillante tolérance dont l'enfant bénéficie jusqu'à trois, quatre, voire cinq ans, s'achève brutalement par un "tour de vis » (crackdown) radical et, affirme-t-il, traumatique. Pour conclure, une idée adressée aux parents indiens: pour changer le système des castes, changez la façon dont votre enfant apprend la propreté.

5 Sans même traiter de la question de fond, qui consiste à vouloir «expliquer » le fonctionnement interactionnel et symbolique complexe d'une société sur quelques milliers d'années par la fixation infantile de ses membres au stade anal, notons que la démonstration ne respecte pas quelques précautions élémentaires. Tout en enfonçant à l'occasion des portes ouvertes (symbolisme des oppositions corporelles droite/ gauche, etc.; p. 61), plusieurs arguments avancés sont des extrapolations risquées: l'auteur suggère par exemple qu'un ancien mythe védique «rendait compte initialement [de l'origine] des intouchables par une partie du corps, en l'occurrence l'anus » (p. 133), alors que ce mythe tel qu'il nous est parvenu ne mentionne rien de tel. Plusieurs affirmations sont de pures projections de (son propre) «bon sens ». Niant le principe de hiérarchie qui dans le monde hindou ordonne l'ensemble des êtres vivants, il affirme : " assurément, cela défie la logique de croire que ce sont les gens en tant que tels qui sont considérés comme intouchables»(p.64). Mentionnant le fait que les hindous orthodoxes prennent un bain et changent de vêtements lorsqu'ils rentrent chez eux après leur travail, il s'étonne: "cela paraît être un comportement irrationnel » (p. 91). Enfin, l'ensemble de la civilisation indienne est réifiée comme dans les pires moments des études du type "culture et personnalité » (les Indiens sont-ils des maniaques du détail ? sont-ils avares ou généreux ?). Du coup, puisqu'il s'agit de caractéristiques générales et intemporelles, les citations des sources sont dépourvues de toute périodisation, régionalisation et contextualisation. Alan Dundes se veut novateur, mais cet aspect de l'ouvrage ne l'est guère !

6 Contrairement à ce qu'il pense, Dundes «n'explique » donc pas le système des castes ni l'intouchabilité. Tout au plus rend-il compte, cette fois à juste titre, de la violence des images dépréciatives attachées aux intouchables et de la charge fantasmatique d'un certain nombre de comportements à leur égard. Pour mettre en lumière cette réalité, la théorie psychanalytique n'était sans doute pas indispensable, la plupart de ces images étant tout à fait explicites. Peut-être se justifie-t-elle cependant davantage vers la fin de l'ouvrage, lorsqu'est abordée l'ambivalence des rapports à la mère (mais il n'est alors plus spécifiquement question d'intouchabilité).

7 Le livre, écrit pour un large public, est vivant et distrayant, et la bibliographie est à la fois riche et originale. L'apport à la réflexion sur la société indienne paraît cependant bien léger malgré la volonté affirmée de problématiser les rapports entre éducation du petit enfant et institutions sociales. 


\section{AUTEUR}

\section{GILLES TARABOUT}

CNRS-EHESS, Centre d'études de l'Inde et de l'Asie du Sud, Paris. 